\title{
ChILE 2017: AMBICIONES, ESTRATEGIAS Y EXPECTATIVAS EN EL ESTRENO DE LAS NUEVAS REGLAS ELECTORALES*
}

\author{
Chile 2017: Ambitions, Strategies and Expectatives at the \\ Onset of the New Electoral Rules
}

\section{SERGIO TORO MAUREIRA}

Universidad de Concepción, Chile

\section{MACARENA VALENZUELA BELTRÁN}

Universidad Diego Portales, Chile

\begin{abstract}
RESUMEN
El 2017 en Chile fue un año distinto en términos políticos y coyunturales. Luego de un periodo de calma institucional, el gobierno debió enfrentar la emergencia de los incendios forestales, los problemas de corrupción en Carabineros y las denuncias de maltrato en dependencias del Servicio Nacional de Menores (SENAME). Sumado a ello, ese año se desarrollaron las elecciones presidenciales, parlamentarias y de consejeros regionales. Para el caso de las elecciones parlamentarias, significó también el estreno de la reforma electoral que reemplazó el sistema binominal por otro con distritos de magnitudes variables. Sostenemos que este cambio de regla electoral provocó tres efectos en los actores políticos: a) cambio de ambiciones en un contexto de incertidumbre, b) nuevas estrategias de trabajo territorial en campañas y c) renovación de las expectativas de nuevos actores tradicionalmente excluidos de la competencia electoral.
\end{abstract}

Palabras clave: Chile, incendios forestales, corrupción policial, maltrato a menores, reforma electoral

\begin{abstract}
2017 was an unusual year for politics in Chile. After a period of institutional stability, the government had to face forest fires, police corruption, and reports of abuses within the National Service for Minors (SENAME). In addition, the years saw presidential, parliamentary, and regional elections. This was also the first time that the new electoral system, which replaced the binominal formula with a more proportional system using a great district magnitude, was used for national elections. We argue that this change in the electoral rule had three effects on political actors: 1) uncertainty about the new rules changed political ambitions; 2) it led to new campaign strategies; 3) it gave new actors who had traditionally been excluded from electoral competition new hope.
\end{abstract}

Keywords: Chile, wildfires, police corruption, electoral reform 


\section{INTRODUCCIÓN}

Este artículo relata los principales eventos políticos, económicos y sociales ocurridos en Chile durante el año 2017. Este periodo fue especialmente importante para el país, pues fue el estreno de la reforma electoral aprobada en el año 2015 que cambió el sistema elección del Congreso desde una fórmula proporcional con 60 distritos y 19 circunscripciones bi-nominales, a otra de 28 distritos y 15 circunscripciones de magnitudes medianas y variables según la población.

Precisamente, el nuevo escenario electoral generó un reordenamiento del paisaje político del país. Las nuevas fuerzas políticas como el Frente Amplio o Evolución Política se fortalecieron electoralmente. En cambio, el bloque oficialista de la Nueva Mayoría sufrió un fuerte revés electoral fruto de la decisión de la Democracia Cristiana de llevar un camino distinto al resto de los partidos que conformaban la coalición. En apretada síntesis, el resultado de este año electoral fue: pérdida del gobierno y menor peso parlamentario de la antigua Nueva Mayoría (del 57\% al 36\% en la Cámara Baja), triunfo en las presidenciales y mayor proporción parlamentaria de Chile Vamos (de 140\% al $47 \%$ ) y la irrupción de una nueva fuerza como el Frente Amplio (del 2,5\% al $13 \%)$.

Este artículo observa las ambiciones, estrategias y expectativas de los actores políticos ante el nuevo escenario. En específico, se argumenta que las nuevas reglas electorales produjeron tres efectos importantes en el sistema político: a) cambio de las ambiciones ante la incertidumbre b) nuevas estrategias de trabajo territorial en campaña y c) renovación de las expectativas de nuevos actores o sectores tradicionales históricamente excluidos de la competencia electoral.

De igual manera, el año 2017 fue el escenario de otros eventos que marcaron la coyuntura económica, social e institucional. Este recuento analizará aquellos que tuvieron repercusión política y motivaron acciones concretas del gobierno. En el área económica los analistas coincidieron en la recuperación del crecimiento y la consolidación del proyecto de diversificación energética del país. En lo social, la coyuntura la marcó la crisis de los incendios forestales desde la región de Valparaíso a La Araucanía, incendios que fueron cuatro veces más grandes que el récord histórico de hectáreas quemadas en país. Finalmente, en lo institucional, el gobierno debió lidiar tanto con el fraude en Carabineros por un monto estimado de U\$S 43 millones, como con la denuncia del diputado Eduardo Saffirio por la muerte injustificada de niños internados en los hogares de menores que funcionaban bajo la supervisión del Servicio Nacional de Menores (SENAME).

En consecuencia, el artículo se divide en cuatro partes. La primera se ocupa de desarrollar en detalle los temas sociales, económicos e institucionales mencionados anteriormente. La segunda parte describe el funcionamiento del Congreso y el Ejecutivo en un año en que el gobierno debió enfrentar sus 
proyectos en un contexto de baja popularidad, con crisis dentro de la coalición y disputas internas en sus partidos. La tercera analiza la implicancia del nuevo sistema electoral y la reconfiguración del sistema de partidos en Chile. El texto finaliza con una discusión sobre las perspectivas futuras del sistema político del país.

\section{TEMAS DE COYUNTURA}

\section{Coyuntura económica: recuperación económica y diversificación energética}

El año 2017 fue un periodo de recuperación de las dificultades de crecimiento que arrastraba la economía chilena desde el 2014. Al igual que buena parte de los países latinoamericanos que dependen de las exportaciones de materias primas, la debilidad del comercio mundial (OECD 2017) produjo que en años anteriores Chile tuviera un descenso de los precios y una reducción de la rentabilidad. Esto se reflejó especialmente en la explotación del cobre —que es fuente importante de ingresos del país-, que hasta el 2016 tuvo una baja sustancial de la inversión y pérdida de empleos mineros. De acuerdo con el informe de estudios económicos de la OCDE (2018), Chile ha experimentado una recuperación reciente, al igual que el resto de los países latinoamericanos. El siguiente gráfico muestra la evolución del PIB de Chile en comparación con América Latina y el Caribe.

Figura 1. Evolución del Producto Interno Bruto (2005-2017)

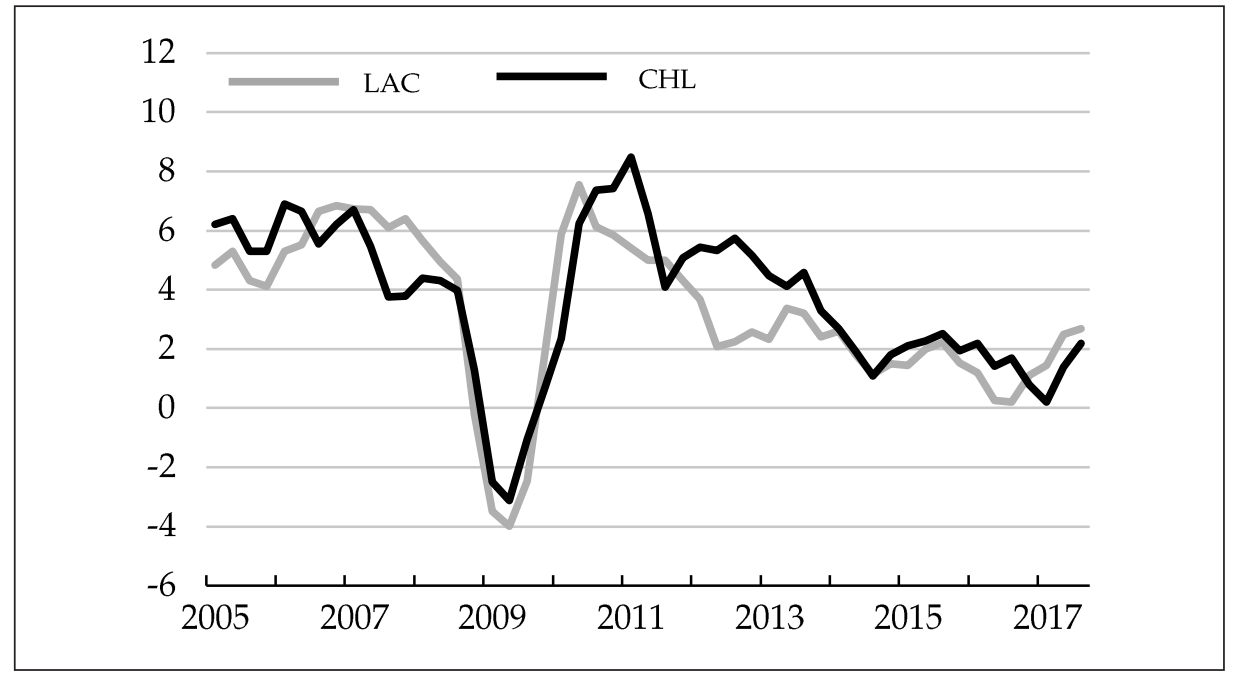

Fuente: OECD 
En lo referido a las tasas de desempleo e inflación, el año estuvo dentro de los parámetros normales. La inflación anual entre enero de 2017 y enero de 2018 fue de 2,19\%. Del mismo modo, el desempleo alcanzó el 6,4\%, 0,3 puntos más que el año anterior. Como siempre, la mayor tasa de desocupación la registraron los jóvenes (15-29 años) con 13,7\%. De todas maneras, las cifras fueron estables y dentro de los parámetros de consolidación del mercado del trabajo. Llama la atención que los empleos que más subieron durante el último trimestre fueron los del rubro enseñanza (72.000 nuevos trabajadores) y de administración pública (29.000 nuevos trabajadores). ${ }^{1}$

Cabe destacar que las tasas de desempleo fueron distintas en cada una de las regiones del país. Por ejemplo, la zona del extremo sur chileno de Magallanes se presentó un índice de un 2.1\%, mientras que La Araucanía, región pobre y con alta población indígena, alcanzó una cifra de 8,8\%.

Figura 2. Tasa de desempleo por región del país 2017

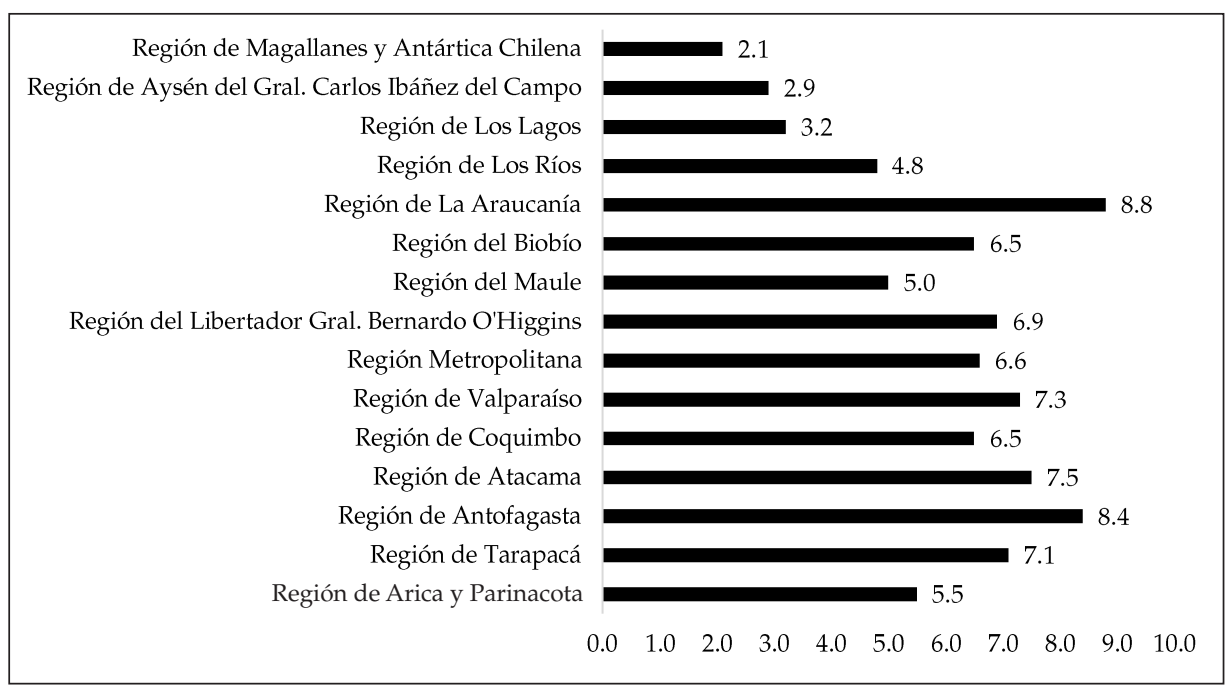

Fuente: Instituto Nacional de Estadística

Por otro lado, el 2017 se registró la consolidación de un proceso de crecimiento en materia de diversificación de la matriz energética. Un hito clave que respalda esta afirmación fue la inauguración de la primera planta geotérmica de América Latina en septiembre de 2017. Si bien es una planta pequeña, con una demanda de consumo anual de 165.000 familias, su inauguración marcó la consolidación de las Energías Renovables No Convencionales (ERNC). En efecto, Chile avanzó fuertemente en la potencia instalada con base en la ERNC. A 2017, esa potencia 
ascendió a 4.110 MW (CNE 2018), cifra que corresponde al 18\% de la capacidad eléctrica total del país. Esto sin duda fue un impulso para la economía del país, pues el desarrollo de energías renovables tiene efectos positivos de colaboración económica por la generación de redes activas de inversión entre gobiernos, planificadores de energía, agencias de cooperación internacional y organismos asociados (Bhattacharya et al. 2016).

Sin embargo, el avance en la ERNC contrastó con los incendios de las plantaciones forestales durante el verano de 2017, acontecimiento que durante más de un mes movilizó los recursos políticos y fiscales del gobierno.

\section{Coyuntura social: Los incendios forestales}

El comienzo del año 2017 estuvo muy lejos de la normalidad. Con un saldo de 7150 personas afectadas, 3324 damnificados y 11 fallecidos, nunca el país había sufrido una oleada de incendios forestales como la que se presentó el mes de enero. Las incipientes investigaciones sobre este acontecimiento consideraron que su irrupción fue producto de una combinación de condiciones relacionadas con la actividad humana, altas temperaturas, vientos secos y extensas sequías atribuidas al cambio climático del antropoceno (Martinez-Harms et al. 2017). Por otro lado, desde los expertos del gobierno, se sostuvo que la razón de lo ocurrido fue la combinación llamada "30-30-30", que es una situación extrema en que se presentan tres condiciones climáticas que en su conjunto permiten la propagación del fuego. Más específicamente, 30 o más grados de temperatura, $30 \%$ o menos de humedad del ambiente y 30 o más nudos de velocidad del viento (Domínguez 2016).

Si bien aún se discuten las verdaderas razones de los incendios forestales, lo cierto es que el 2017 fue extraordinario en cuanto a hectáreas quemadas. El gráfico siguiente muestra la evolución de las hectáreas afectadas en los últimos 33 años. 
Figura 3. Hectáreas afectadas por temporada (1984-2017)

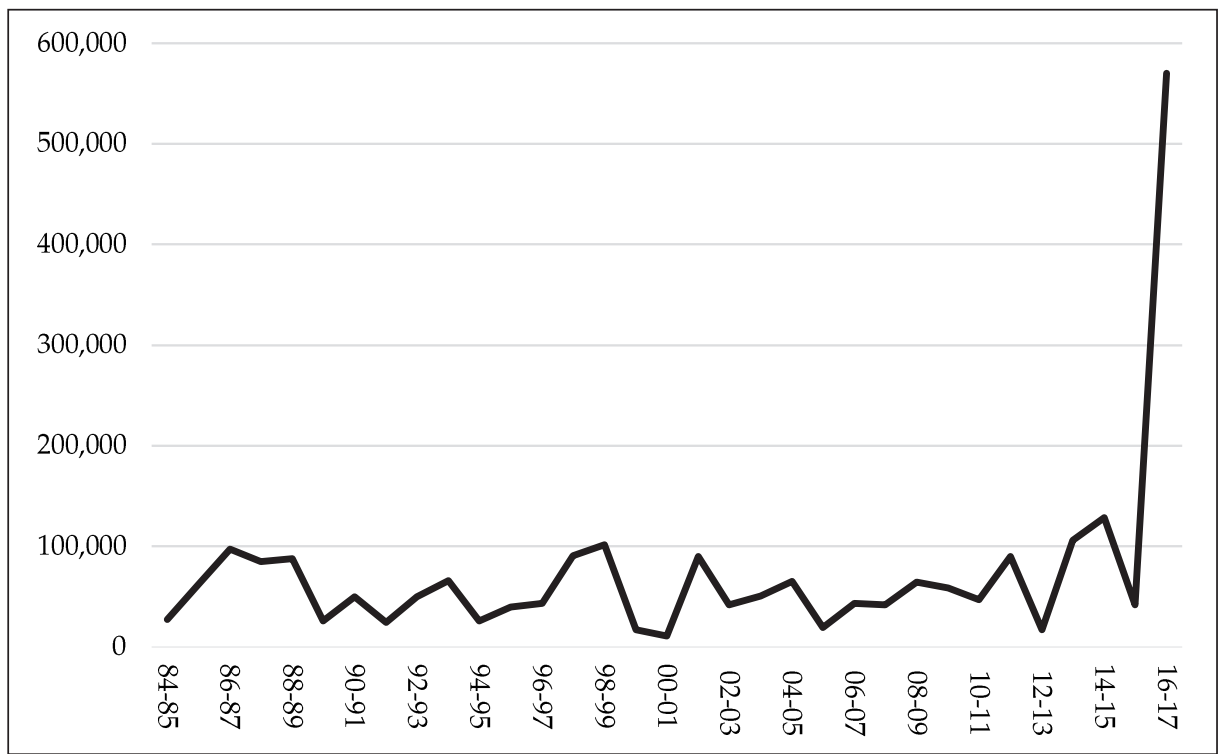

Fuente: CONAF

El manejo de monocultivo también ayudó a la rápida propagación del incendio. Las zonas con más actividad incendiaria fueron precisamente la regiones del Maule (VII) y Biobío (VIII), ambas en especial durante el mes de enero.

Figura 4. Hectáreas consumidas por mes y región

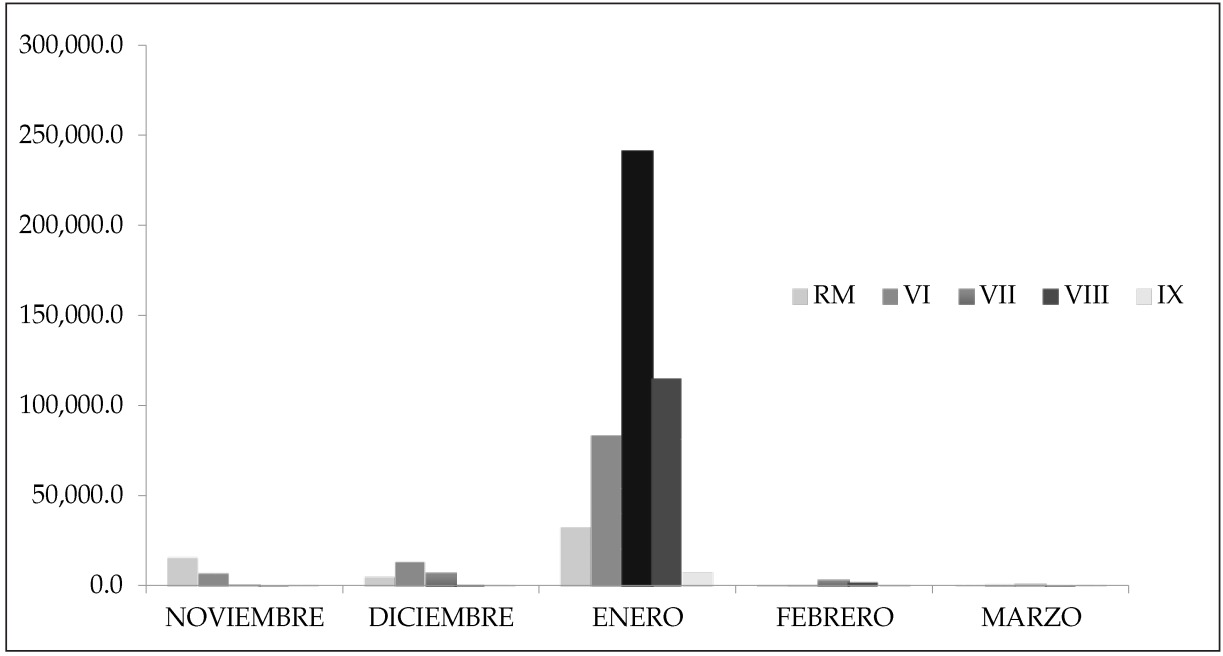

Fuente: CONAF 
Estas regiones se han enfrentado a una industria indiscriminada de plantación forestal que desplazó al bosque nativo desde la década de los ochenta. Es más, la esperada ley de "recuperación del bosque nativo y de fomento forestal", que fue presentada en el año 1992, demoró 16 años en ser aprobada justamente para que las empresas desarrollaran las talas e inversiones en zonas como los valles de la Cordillera de la Costa y la cordillera de Nahuelbuta (Boeninger 2007). Además, el último catastro de CONAF confirma que en la región del Maule, el $60 \%$ de los bosques es plantación forestal, mientras que en la región del Biobío este tipo plantación corresponde al 58\%.

Fue precisamente en los lugares asediados por plantaciones forestales donde se concentró la mayor parte de las hectáreas quemadas. Poblados como Nivirilo y Santa Olga fueron los más afectados. Este último, con cerca de 3.000 habitantes, desapareció totalmente por efecto del fuego. El gobierno nombró a Sergio Galilea en la labor de reconstrucción, trabajo que demandó ejes de vivienda, infraestructura pública, reforestación, energía, salud, entre otros (Gobierno de Chile 2017). Sensores térmicos de la NASA originados de los espectrorradiómetro de satélites muestran los lugares del desastre.

Figura 5. Zonas de incendio en la Región del Maule (izq.) y Biobío (der.)
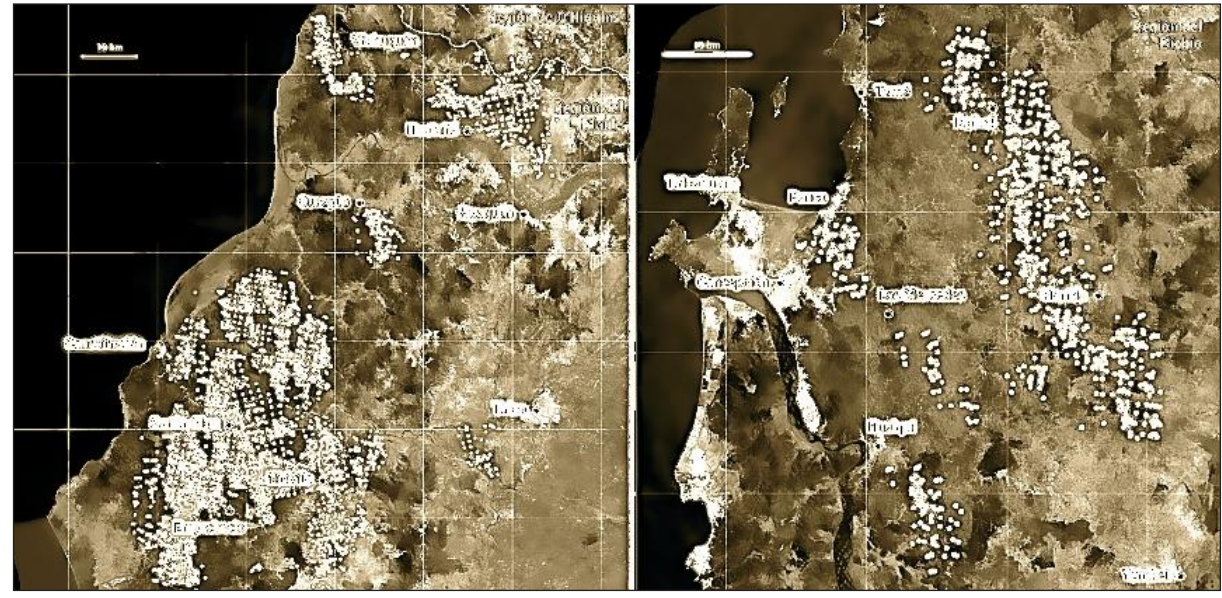

Zonas destacadas son zonas de incendio.

Fuente: Plataforma Urbana (2017)

No obstante, esta no fue la única crisis que debió sortear el gobierno de Bachelet. Desde varios años se venían gestando dos crisis institucionales. La primera es la crisis de Carabineros producto del desfalco de 26 mil millones de pesos. La segunda es la muerte de niños y niñas bajo tutela del Estado en los centros del SENAME. 


\section{Coyuntura institucional: Las crisis de Carabineros y del SENAME}

a) La crisis de Carabineros

Una de las crisis institucionales que marcó la agenda política del año 2017 fue el caso de Carabineros de Chile. Esta crisis tuvo dos aristas: el desfalco al fisco por parte de la institución y las pruebas falsas creadas y utilizadas por la inteligencia de Carabineros para culpar a comuneros mapuches de violencia política (Operación Huracán). Esta crisis no sólo involucró a la misma institución armada, sino que además arrastró a la Agencia Nacional de Inteligencia, al Ministerio del Interior y al Ministerio Público. Más allá de comprometer a otras instituciones, el principal problema fue que se enfrentaron dos instituciones constitutivas del engranaje del sistema procesal penal del país: Carabineros y la Fiscalía.

Tres son las etapas que estructuraron la crisis. La primera se constituye por el desfalco de dinero y el desarrollo de las investigaciones por parte de la fiscalía de Magallanes para esclarecer los hechos. En segundo lugar, la denuncia de las pruebas falsas creadas por carabineros e imputadas a los comuneros mapuches. Finalmente, la conjunción de ambas irregularidades condicionó la renuncia del alto mando y repercusiones en la opinión pública.

Respecto del primer caso, en noviembre de 2017 el fiscal logró 130 formalizados entre oficiales, exoficiales, civiles y contratados por resolución en la institución de Carabineros. Se descubrió una cofradía compuesta por los más altos mandos de la institución denominada "la mafia de la intendencia". A la fecha de la redacción de este artículo, el fraude ha involucrado más de 26 mil millones de pesos malversados a través de tres mecanismos: i) el manejo de los fondos de remuneraciones, ii) los fondos de desahucio y iii) los fondos de asignaciones reservadas (Sepúlveda y González 2018)

El mismo año, específicamente en septiembre, la "Operación Huracán" (investigación dirigida por Carabineros bajo la Ley de Inteligencia) detuvo a ocho comuneros mapuche relacionados con la Coordinadora Arauco Malleco (CAM) —organización mapuche radical- acusándolos de tráfico de armas desde Argentina. De acuerdo con la fiscalía, estas armas habrían sido utilizadas en la quema de iglesias y camiones en la zona de La Araucanía (IX región). Las pruebas para dicho arresto se basaron en la existencia de conversaciones de Whatsapp y Telegram (Sepúlveda y González 2018). Sin embargo, no tardó mucho en advertirse el montaje de Carabineros. Es así como la fiscalía descubrió irregularidades entre los informes y los hallazgos efectivos. Como resultado, el 28 de diciembre del 2017 la unidad especializada de la fiscalía entregó un documento en el que se indicaba que los informes de la policía uniformada habían sido manipulados con pruebas falsas. Así, se ordena el allanamiento de las dependencias de la Unidad de Inteligencia Operativa Especializada (UIOE) de Temuco (Sepúlveda y González 2018) y se determina la responsabilidad de 
nueve carabineros y un civil por la alteración de pruebas y obstrucción de la justicia.

Finalmente, en medio de la crisis, el Director General de Carabineros viajó de vacaciones a Miami. El gobierno, consternado por tal decisión, solicitó su retorno a la brevedad. El 12 de marzo de 2018, Bruno Villalobos renunció a su cargo y asumió como máxima autoridad responsable el Subdirector General de Carabineros, Julio Pineda.

\section{b) Crisis en el Servicio Nacional de Menores (SENAME)}

Otra de las crisis que afectaron la institucionalidad chilena fue el caso de los niños atendidos por el SENAME. Todo comenzó con los resultados del cuestionario que aplicó la jueza Mónica Jeldres en el año 2012. Estos resultados arrojaron que 12 niños, de 400 que participaron en el estudio, fueron abusados sexualmente. Este primer resultado de las investigaciones abrió la puerta para acceder a la revisión de los antecedentes de 6.500 niños, así como al examen de las condiciones materiales en 108 residencias.

Los resultados de tales pericias expusieron como resultado "grave" los problemas de abuso sexual, educación y calidad de vida. Ello sumado a niños con enfermedades crónicas y de salud mental sin tratar, uso y abusos de fármacos, falencias en el trabajo con las familias y existencia de una red de explotación sexual de menores en uno de los hogares. Esta investigación, conocida como "Comisión Jeldres", enfatizó la importancia del rol del juez en el seguimiento de los casos de menores y exigió que se garantizaran los derechos del niño (Cámara de Diputados 2013). Todos estos hallazgos fueron reforzados cuando una niña de 11 años, llamada Lissette Vega, falleció en el interior de un centro de protección del SENAME. Este hecho impulsó mayores investigaciones sobre la institución a cargo de los menores. En primer lugar, se creó la comisión investigadora SENAME para analizar los casos de muertes dentro de la institución. Los resultados arrojaron que 1.313 niños habrían muerto bajo la tutela del Servicio (Saffirio 2017).

Ante ello, Marcela Labraña renunció como directora y asumió Hugo Herrera. Sin embargo, unos días después que la fiscalía anunciase los resultados de investigación, Herrera renunció también a su cargo. La ministra de Justicia Javiera Blanco nombró a Solange Huerta en su reemplazo. En octubre de 2017, la entonces presidenta Michelle Bachelet anunció la salida de Javiera Blanco del Ministerio de Justicia y designó en su lugar a Jaime Campos. Las investigaciones realizadas por la comisión de investigación de la Cámara arrojaron un reproche a la ministra Blanco por su "inexcusable negligencia" y por sus actos de omisión al enfrentar la crisis (Cámara de Diputados 2017). 


\section{LOS PODERES EJECUTIVO Y LEGISLATIVO}

\section{El Poder Ejecutivo: la tensión en el equipo económico}

Los cambios ministeriales pueden ser un síntoma de inestabilidad de los gobiernos (Martínez-Gallardo 2012). En algunas ocasiones un cambio, por mínimo que sea, puede ser una señal de dificultades de los presidentes. Así, la rotación ministerial ayudaría a los gobiernos a generar los ajustes necesarios en tiempos de crisis. Autores como Camerlo y Pérez Liñán (2014) indican que los Ejecutivos definen sus ministros para fortalecer el control sobre el gabinete. De esta forma actúan preservando a los tecnócratas responsables de las políticas exitosas y en menor medida a los actores políticos leales.

Por ello las renuncias unilaterales de ministros en sistemas presidenciales fuertes como el chileno tienen una connotación de crisis. Esto es justamente lo que ocurrió a fines de agosto de 2017 con la complicada relación de Bachelet con su equipo económico. En efecto, a la tensión permanente con el ministro Hacienda Rodrigo Valdés, se le sumó un desencuentro con el titular de economía, Felipe Céspedes, tensión que terminó en la renuncia de todo el equipo.

El conflicto comenzó con el rechazo en votación dividida realizado por el Comité de Ministros para la Sustentabilidad del proyecto minero portuario Dominga, por no contar con las suficientes medidas de mitigación ambiental. Este rechazo del comité, que era dirigido por el entonces titular de Medio Ambiente Marcelo Mena, se vio enfrascado en una polémica cuando Céspedes decidió no participar porque los antecedentes fueron entregados con poco plazo de antelación. Estas declaraciones fueron inmediatamente desmentidas por Mena al señalar que los "antecedentes para tomar una decisión existían" y que "cinco de los seis ministros del comité emitieron un pronunciamiento respecto del proyecto" (Albertini 2017).

La declaración del ministro Mena no fue bien recibida por el comité económico. Es así como comenzó una guerra de declaraciones que involucró, por un lado, al ministro y subsecretario de Hacienda (Rodrigo Valdés y Alejandro Micco) y, por el otro, a la vocera de Gobierno y al subsecretario del Interior (Paula Narváez y Mahmud Aleuy). Ante la disputa, Narváez mantuvo una posición sobre el respeto de la institucionalidad, cuando señaló: "Tenemos la obligación de no solo respaldar la institucionalidad que tenemos sino que tenemos la obligación de acatar, por eso somos funcionarios públicos" (Tele13 2017). Esta posición fue respondida por Valdés de manera amenazante, cuando afirmó que algunos no tenían "el crecimiento entre las prioridades". Así, el conflicto escaló a tal punto que fue la propia presidenta quien zanjó el tema avalando la decisión del Comité de Ministros. Al respecto, Bachelet sostuvo que "Chile necesita que su crecimiento vaya de la mano del cuidado del medio ambiente". 
Un par de días después, dos ministros y un subsecretario del equipo económico presentaron su renuncia. En el caso de los ministros fueron reemplazados por "viejos conocidos", Nicolás Eyzaguirre en Hacienda y Jorge Rodríguez Grossi en Economía. Las tablas siguientes dan cuenta de los cambios en Ministerios y Subsecretarías 2017.

Tabla 1. Cambios de Ministros(as) 2017

\begin{tabular}{|c|c|c|c|}
\hline $\begin{array}{l}\text { FECHA DE } \\
\text { INGRESO }\end{array}$ & MinisteRIO & $\begin{array}{l}\text { Ministro(A) } \\
\text { ENTRANTE }\end{array}$ & $\begin{array}{l}\text { Ministro(A) } \\
\text { SALIENTE }\end{array}$ \\
\hline $\begin{array}{l}31 \text { de agosto } \\
\text { de } 2017\end{array}$ & Hacienda & $\begin{array}{l}\text { Nicolás Eyzaguirre } \\
\text { Guzmán }\end{array}$ & $\begin{array}{l}\text { Rodrigo Valdés } \\
\text { Pulido }\end{array}$ \\
\hline $\begin{array}{l}31 \text { de agosto } \\
\text { de } 2017\end{array}$ & $\begin{array}{l}\text { Secretaría General de } \\
\text { la Presidencia }\end{array}$ & $\begin{array}{l}\text { Gabriel de la Fuente } \\
\text { Acuña }\end{array}$ & $\begin{array}{l}\text { Nicolás Eyzaguirre } \\
\text { Guzmán }\end{array}$ \\
\hline $\begin{array}{l}31 \text { de agosto } \\
\text { de } 2017\end{array}$ & $\begin{array}{l}\text { Economía, Fomento } \\
\text { y Turismo }\end{array}$ & $\begin{array}{l}\text { Jorge Rodríguez } \\
\text { Grossi }\end{array}$ & $\begin{array}{l}\text { Luis Felipe Céspedes } \\
\text { Cifuentes }\end{array}$ \\
\hline $\begin{array}{l}14 \text { de marzo } \\
\text { de } 2017\end{array}$ & $\begin{array}{l}\text { Transporte y } \\
\text { Telecomunicaciones }\end{array}$ & Paola Tapia Salas & $\begin{array}{l}\text { Andrés Gómez-Lobo } \\
\text { Echenique }\end{array}$ \\
\hline $\begin{array}{l}20 \text { de marzo } \\
\text { de } 2017\end{array}$ & Medio Ambiente & $\begin{array}{l}\text { Marcelo Mena } \\
\text { Carrasco }\end{array}$ & $\begin{array}{l}\text { Pablo Badenier } \\
\text { Martínez }\end{array}$ \\
\hline
\end{tabular}

Tabla 2. Cambios de Subsecretarios(as) 2017

\begin{tabular}{llll}
\hline $\begin{array}{l}\text { Fecha de } \\
\text { Ingreso }\end{array}$ & Subsecretaría & $\begin{array}{l}\text { Subsecretario(A) } \\
\text { Entrante }\end{array}$ & $\begin{array}{l}\text { Subsecretario(A) } \\
\text { SAliente }\end{array}$ \\
\hline $\begin{array}{l}5 \text { de } \\
\text { septiembre } \\
\text { de } 2017\end{array}$ & Hacienda & $\begin{array}{l}\text { Macarena Lobos } \\
\text { Palacios }\end{array}$ & $\begin{array}{l}\text { Alejandro Micco } \\
\text { Aguayo }\end{array}$ \\
$\begin{array}{l}5 \text { de } \\
\text { septiembre } \\
\text { de } 2017\end{array}$ & Secretaría General de & $\begin{array}{l}\text { Víctor Maldonado } \\
\text { Roldán }\end{array}$ & $\begin{array}{l}\text { Gabriel de la Fuente } \\
\text { Acuña }\end{array}$ \\
$\begin{array}{l}10 \text { de abril } \\
\text { de } 2017\end{array}$ & Pesca y Acuicultura & $\begin{array}{l}\text { Pablo Berazaluce } \\
\text { Maturana }\end{array}$ & $\begin{array}{l}\text { Raúl Súnico } \\
\text { Galdámez }\end{array}$ \\
$\begin{array}{l}\text { de } 2017 \\
17 \text { de mayo } \\
\text { de } 2017\end{array}$ & Medio Ambiente & $\begin{array}{l}\text { Cristián Gutiérrez } \\
\text { Pangui }\end{array}$ & $\begin{array}{l}\text { Marcelo Mena } \\
\text { Carrasco }\end{array}$ \\
\hline
\end{tabular}




\section{Poder Legislativo}

Desde hace un buen tiempo que el Congreso Nacional tiene un funcionamiento normal y constante en cuanto su rol legislativo. Desde el año 2006, la presentación de proyectos de ley (mociones) se ha normalizado en un promedio aproximado de 500 presentaciones, cifra superior comparada con los años noventa, en que estas bordeaban un promedio de 100. Las explicaciones de esa tendencia son dos. La primera es la práctica del Ejecutivo de considerar las iniciativas de los parlamentarios como antecedentes importantes en la presentación de los proyectos de ley, práctica que entrega mayor visibilidad a los parlamentarios cuando se discuten iniciativas del gobierno. En sintonía con la anterior, la segunda explicación se relaciona con el aprendizaje de los parlamentarios en la funcionalidad de las propuestas legislativas, que, si bien no presentan un destino de política, ayudan al posicionamiento de los congresistas en determinados temas de interés (Alemán 2008).

En cuanto a la presentación de iniciativas legislativas, cabe destacar que el año 2017 fue el periodo con menos propuestas por parte de los parlamentarios. Esta disminución tiene relación con el periodo electoral, momento en que los parlamentarios se ocupan más de generar acciones en los distritos que dentro del Congreso (Toro 2017). Continuando con la secuencia del año anterior de este anuario, durante el periodo analizado se observa una alta presentación de iniciativas provenientes de los legisladores, aunque bajo nivel de aprobación de sus iniciativas.

Tabla 3. Mociones y Mensajes presentados período 2014-2017

\begin{tabular}{llll}
\hline 2014 & 2015 & 2016 & 2017 \\
\hline$(\%) \mathrm{N}$ & $(\%) \mathrm{N}$ & $(\%) \mathrm{N}$ & $(\%) \mathrm{N}$ \\
\hline
\end{tabular}

\begin{tabular}{lcccc}
\hline \multicolumn{5}{c}{ Proyectos Ingresados } \\
\hline Moción & $442(82.8 \%)$ & $531(83.1 \%)$ & $504(89.5 \%)$ & $414(85.9 \%)$ \\
Mensaje & $92(17.2 \%)$ & $108(16.9 \%)$ & $59(10.5 \%)$ & $68(14.1 \%)$ \\
Total & 534 & 639 & 563 & 482 \\
\hline \multicolumn{5}{c}{ LEYEs PUbLICADAS } \\
\hline Moción & $41(36 \%)$ & $42(38.5 \%)$ & $45(41.3 \%)$ & $39(40.6 \%)$ \\
Mensaje & $73(64 \%)$ & $67(61.5 \%)$ & $64(58.7 \%)$ & $57(50.4 \%)$ \\
Total & 114 & 109 & 109 & 96 \\
\hline
\end{tabular}


Dentro del Poder Legislativo también cabe destacar el rol fiscalizador de los parlamentarios. Esta función se cumple de distintas maneras. Las más relevantes son las comisiones investigadoras y los oficios de fiscalización.

Sobre las primeras, existieron 10 comisiones investigadoras en temas diversos. Destacan entre ellas las de "Irregularidades en la gestión y administración financiera en Carabineros de Chile" y la de los "Problemas de recursos, logística y organización para manejo de emergencias por incendios forestales" (Cámara de Diputados 2018). Ambas comisiones se dedicaron precisamente a trabajar sobre los temas de crisis institucionales mencionadas más arriba.

Finalmente, respecto de los oficios de fiscalización, durante el año analizado se despacharon 636, de los cuales 119 fueron generados en Sala (principalmente mediante la instancia de hora de incidentes), ${ }^{2} 516$ por secretaría y 1 de solicitud de antecedentes. Sobre los oficios existe una incipiente literatura respecto de su función (Aleman, Micozzi y Ramírez 2016). Se coincide, sin embargo, que uno de los objetivos principales es tratar temas del propio distrito con el fin de tomar posición o reclamar créditos ante determinados requerimientos del electorado. Esta es la razón que explica que el destino de buena parte de los oficios sean las propias municipalidades.

\section{Relación Ejecutivo-Legislativo}

En gran parte de las investigaciones sobre la relación Ejecutivo-Legislativo de los gobiernos posautoritarios, se ha demostrado que los proyectos emanados desde el presidente alcanzan tasas de éxito importantes producto de la capacidad de los gobiernos para coordinar los partidos de la coalición oficialista y llegar a acuerdos específicos con parlamentarios de otro pacto. Desde el gobierno de Ricardo Lagos (Toro 2007) hasta el de Sebastián Piñera (Campos-Parra y Navia 2017; Toloza y Toro 2017), las investigaciones han mostrado alta disciplina en los partidos de gobierno y menor disciplina en los de oposición.

Si bien no existe investigación sobre la conducta parlamentaria durante el segundo periodo de Bachelet, la aprobación de proyectos emblemáticos del gobierno hace suponer que el comportamiento no dista mucho de lo que ha estado sucediendo desde hace 27 años. En este artículo nos centraremos en dos: el proyecto que "regula la despenalización de la interrupción voluntaria del embarazo en tres causales" (Boletín 9.895-11) y la iniciativa "sobre educación superior" (Boletín 10.783-04).

Ambas iniciativas tienen su origen en años anteriores pero fueron activadas con fuerza durante 2017. La primera -la de interrupción del embarazoalcanzó 25 urgencias, siendo las siete últimas de suma urgencia. Este proyecto es precisamente un ejemplo de coordinación de los partidos de gobierno y la

2 Al respecto ver Aleman, Micozzi y Ramírez (2016). 
búsqueda de acuerdos con la oposición. En efecto, si se considera la última votación relacionada con el despacho de la Comisión Mixta es posible constatar que, a pesar de que los partidos de la Nueva Mayoría se encontraban en medio de una disputa por la inscripción de las listas de los candidatos parlamentarios y presidenciales, todos los representantes de las bancadas oficialistas votaron cohesionadamente a favor de la iniciativa, sumando además algunos votos de Renovación Nacional — como los de Karla Rubilar y Joaquín Godoy— además de otros partidos o parlamentarios independientes. El resultado final fue 70 votos a favor, 45 en contra y 1 abstención.

Un segundo ejemplo es el proyecto de educación superior con Quórum de Ley Orgánica Constitucional. Solo en la Cámara de Diputados, este proyecto tuvo 157 votaciones en sala. Una de las fechas de mayor intensidad en la votación fue el 17 de junio de 2017, cuando buena parte de los artículos fueron votados "en particular". Al igual que el caso anterior, la iniciativa necesitó de la unidad de los parlamentarios de la Nueva Mayoría y algunas conversaciones con diputados que no eran parte de la coalición de gobierno (como el caso de Alejandra Sepúlveda o Vlado Mirosevic). Un ejemplo es la votación del artículo 1 del proyecto, que se aprobó con 70 votos a favor y 41 votos en contra.

En consecuencia, la fórmula del éxito de estos proyectos tuvo la misma receta que las mismas administraciones, receta que podría cambiar o mantenerse con la nueva conformación del Congreso.

\section{EL PROCESO ELECCIONARIO}

Durante 2017 tuvieron lugar tres fechas eleccionarias que terminaron con Sebastián Piñera como presidente de la República. En esas tres fechas se decidieron los candidatos presidenciales y algunos candidatos al Congreso de Chile Vamos y el Frente Amplio (2 de julio), la composición de la Cámara de Diputados, la mitad del Senado y la primera ronda presidencial (19 de noviembre) y la definición presidencial en segunda ronda (17 de diciembre). A continuación se realizará un relato detallado de este proceso.

\section{Las primarias}

El 2 de julio de 2017 fue la primera de estas tres fechas eleccionarias con la primaria presidencial. Esta primaria fue aprovechada solo por dos bloques políticos: Chile Vamos y el Frente Amplio. En efecto, los partidos de la Nueva Mayoría no llegaron a presentarse en las primarias legales producto del conflicto germinado por la decisión del Partido Socialista de no apoyar a Ricardo Lagos e inclinarse por el candidato independiente Alejandro Guillier, así como la decisión del Partido Demócrata Cristiano de tener como candidata propia a Carolina Goic. 
La primaria de Chile Vamos fue sin duda la más bullada y mediática. Tres candidatos se presentaron en esta primaria: Felipe Kast, Sebastián Piñera y Manuel José Ossandón. Este último se enfrentó al expresidente Sebastián Piñera con una estrategia confrontacional que buscaba resaltar sus problemas con la justicia durante el régimen militar. En un debate televisivo y tras una interpelación de Piñera, Ossandón replicó con una de las frases más famosas de la campaña: "Yo fui gerente de una empresa agrícola, él fue gerente del Banco de Talca. Pero según un video que vi de la señora Mónica Madariaga, estuvo escondido porque fue declarado reo. No lo declararon reo por lindo y ha estado metido en un montón de problemas que siempre le echa la culpa a uno más chico" (El Mostrador 2017). Las redes sociales festinaron con el hashtag \#NoTeDeclararonReoPorLindo al punto que Ossandón incrementó sus apariciones televisivas de manera exponencial.

En tanto, Piñera tuvo su momento en esta disputa cuando la periodista Pilar Molina denunció la triangulación de boletas por parte de Ossandón para pagar a su hermana con cargo a la Municipalidad de Pirque cuando él ejercía como Alcalde. Si bien el precandidato inmediatamente culpó al comando del expresidente, esa acusación fue desmentida haciendo una separación discursiva entre las mentiras de Ossandón y el tono conciliador de su postura. Ante las acusaciones de intervención, Piñera respondió: “...y las mentiras son más dolorosas cuando el que las dice sabe que está mintiendo. Pero yo me he hecho el firme propósito de no descalificar a mis compañeros de pacto, ni tampoco a los adversarios..." (24 horas 2017c).

Un tercer actor de estas primarias fue el exministro de Desarrollo Social de Piñera, Felipe Kast. Producto de la estrategia de Ossandón, su comportamiento estuvo alejado de las controversias mediáticas tratando de articular las futuras candidaturas al Congreso de su partido Evolución Política (Evópoli). Es así como sus actividades se concentraron en zonas donde luego se podrían negociar cupos parlamentarios para el partido.

Con todo, los resultados de la primaria de Chile Vamos dieron por ganador a Sebastián Piñera en casi todas las comunas del país. Sin embargo, Ossandón mantuvo las llaves de la zona sur oriente de la Región Metropolitana ganando las comunas de La Florida, Lo Espejo, Pedro Aguirre Cerda, San Joaquín, San Ramón, Pirque, Puente Alto, San José de Maipo, La Granja y La Pintana. Este resultado fue la herramienta de negociación para que Piñera considerara el poder político del senador para sus pretensiones de triunfo en la segunda vuelta. En la siguiente figura se muestran las comunas en las que ganó Ossandón, así como el resultado a nivel nacional. 
Figura 6. Comunas en que ganó Ossandón y resultados finales de las primarias de Chile Vamos

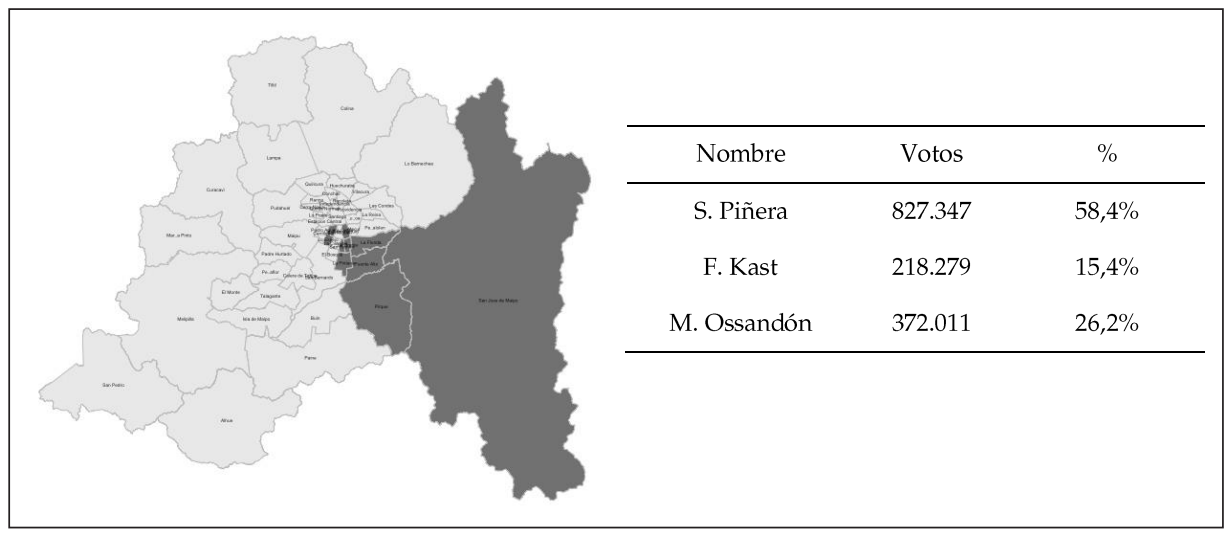

En gris oscuro las comunas en que ganó Manuel José Ossandón. En gris claro, aquellas que ganó Sebastián Piñera. Felipe Kast no obtuvo triunfo en ninguna comuna de la región Metropolitana de Santiago.

Al otro lado de la vereda, la primaria del Frente Amplio fue algo más amistosa aunque no exenta de problemas. Revolución Democrática y una serie de movimientos que conformaban la coalición propusieron como abanderada a la periodista de la Universidad de Concepción, Beatriz Sánchez. Sánchez, que ya tenía una dilatada trayectoria como periodista, recorrió buena parte del país buscando firmas para su partido, así como también tratando de posicionar su candidatura. Al frente, encontró al sociólogo Alberto Mayol. Con un discurso más radical que postulaba el derrumbe del modelo, Mayol participó en los diferentes foros dando un contrapunto más contestatario y de izquierda. Finalmente, triunfó sin contratiempos la opción de Beatriz Sánchez con una diferencia de más del doble de votos con respecto a Mayol.

Tabla 4. Resultados finales de las primarias del Frente Amplio

\begin{tabular}{ccc}
\hline Nomвre & Voтоs & $\%$ \\
\hline B. Sánchez & 221.348 & $67,6 \%$ \\
A. Mayol & 106.265 & $32,4 \%$ \\
\hline
\end{tabular}

Fuente: www.servel.cl

\section{Las elecciones parlamentarias: ambiciones, estrategias y expectativas}

Las elecciones parlamentarias fueron la prueba de fuego para el funcionamiento de la nueva ley electoral (Ley 20.840), que sustituyó el sistema binominal por uno más proporcional al ampliar las magnitudes distritales y la constitución de las listas. Aprobada el 5 de mayo de 2015, esta ley provocó cambios importantes en 
materia de dibujo distrital, magnitud de distrito y equidad de género (Gamboa y Segovia 2016).

En cuanto al dibujo distrital, la ley contempló la creación de 28 distritos y 15 circunscripciones senatoriales. En el caso del Senado, ese panorama fue adecuado a la división administrativa del país. En tanto, los diseños distritales fueron producto o de la fusión de dos o más distritos antiguos o de un nuevo dibujo atendiendo la densidad poblacional y los intereses políticos subyacentes. En el mismo punto, los distritos de las zonas extremas, es decir, los antiguos 1, 2, 59, 60, mantuvieron sus límites geográficos aunque cambiaron de magnitud de dos a tres escaños.

En efecto, uno de los cambios más importantes de la reforma fue cambiar la magnitud de los distritos. En el caso de los diputados, este cambio de reglas constituyó magnitudes de distrito variables que fluctuaron desde los tres escaños en las zonas extremas hasta ocho en las zonas más densamente pobladas. La regla general de definición de las magnitudes fue la cantidad de escaños obtenida por la suma de la fusión de los distritos binominales +1 . $^{3}$ Para Altman (2016), la distribución de las magnitudes tendió a favorecer al centro metropolitano y menos a las provincias ubicadas en el centro norte y sur del país. De igual forma, observó una pérdida de poder real de las zonas más pobres de Chile (Altman 2016). En el caso de las circunscripciones senatoriales la fórmula fue algo distinta. En varios territorios se mantuvo la elección de dos escaños, mientras otras se elevaron a tres. La región de Valparaíso, Santiago, Biobío y La Araucanía, regiones anteriormente divididas en dos circunscripciones, quedaron con 5 escaños. En total, la ley definió 155 escaños para la Cámara de Diputados y 50 escaños para el Senado.

Respecto del tema de género, por primera vez se estableció una banda de paridad para la inscripción de candidaturas. La ley estableció que de la totalidad de las candidaturas declaradas por los partidos políticos, debía existir una relación 60/40 en el equilibrio de hombres y mujeres. La normativa también estableció una sanción por el no cumplimiento de esta norma señalando que ello acarrearía el rechazo de "todas las candidaturas declaradas a diputados o a senadores, según corresponda, del partido que no haya cumplido con este requisito" (Ley 20840 2015)

Con estas nuevas reglas del juego, los distintos sectores políticos inscribieron sus candidatos y luego comenzaron la campaña. La relación de las inscripciones entre hombres y mujeres fue de 78/54 para el Senado y 563/397 para Diputados (PNUD 2017). Por supuesto, como todo cambio institucional, las nuevas reglas del juego cambiaron tanto las ambiciones, estrategias y expectativas de los actores involucrados como la configuración de ganadores y perdedores. 


\section{a) Ambiciones, estrategias y expectativas}

Sobre el cambio de ambiciones, los actores políticos incumbentes tomaron una decisión entre tres posibles caminos: a) retirarse de la competencia (ambición discreta), b) competir por la reelección (ambición estática) o c) competir por el Senado (ambición progresiva). ${ }^{4}$ En efecto, el cambio del sistema electoral produjo que al menos 17 diputados decidieran competir al Senado y que otros 19 no volvieran a postular. Es más, para el año 2017 solo 84 diputados (79\%) buscaron la reelección, cifra más baja que los anteriores períodos en que aproximadamente el 90\% volvía a postular al cargo. Además, de los que repostularon, el 76,2\% fue reelecto, solo cinco puntos menos que en 2001, año más bajo de reelección de incumbentes con el anterior sistema.

Sobre las estrategias para hacer frente a distritos más grandes con el nuevo sistema electoral es posible observar al menos una estrategia exitosa tanto de incumbentes como de aspirantes: la segmentación territorial. La dificultad de recursos y el despliegue en distritos más grandes acentuaron la segmentación no sólo en el vínculo personalista (Luna 2016) sino que en el reparto de sectores entre compañeros de lista.

Sobre el mantenimiento del vínculo personalista, sorprenden las continuidades más que los cambios. Por ejemplo, en distritos urbanos como el 20 o rurales como el 19, se mantuvo el personalismo de siempre, ejemplificado en dos diputados que, a pesar de pertenecer al partido más golpeado de la última elección (PDC), se ha mantenido desde 1989 (José Miguel Ortiz) o lleva cuatro períodos, más aquellos ejercidos por el padre (Jorge Sabag).

De igual manera, es posible encontrar un reparto electoral basado en capturas de los territorios. Sólo como ejemplo didáctico se puede observar nuevamente el distrito 20 y constatar cómo las votaciones de cada comuna no fueron homogéneas, sino que dependieron de la capacidad territorial del candidato. En nuestro ejemplo, es visible la diferencia de votos entre distintos territorios, pues en un mismo distrito, el candidato Gastón Saavedra obtuvo 4.191 en Concepción y 13.366 en Talcahuano, cifra muy opuesta a José Miguel Ortiz, quien recibió 14.191 en Concepción y apenas 1.511 en Talcahuano. Este caso es posible de ser observado en diversos distritos - como el de Claudia Mix en Maipú- y puede llegar a ser una buena línea de trabajo para futuras investigaciones.

Finalmente, en términos de expectativas, no cabe duda de que el nuevo sistema electoral abrió la puerta para nuevas propuestas políticas que habían estado invisibilizadas por el sistema binominal. Se consiguió, por ejemplo, que consejeros regionales y alcaldes — que anteriormente estaban limitados en sus expectativas - alcanzaran un cupo parlamentario, que más mujeres fueran a la competencia política y que nuevos partidos o movimientos —además de partidos que habían sido relegados de la competencia- se transformaran en 
alternativas políticas viables. Todo esto nos lleva a la segunda afirmación sobre quiénes perdieron, ganaron o se mantuvieron ante el cambio.

b) Quiénes perdieron, ganaron o se mantuvieron.

Con la nueva conformación del Congreso, algunos partidos políticos tradicionales perdieron escaños valiosos, principalmente por no llegar a buenos acuerdos en la conformación de las listas. Un ejemplo claro es la Democracia Cristiana, cuyo camino propio hizo que seis de sus parlamentarios perdieran y que, como consecuencia, afectaran el éxito de la lista de sus antiguos socios de la Nueva Mayoría.

Un recuento de cuántos parlamentarios que se presentaron a la reelección no alcanzaron los votos necesarios muestra que 16 de 20 incumbentes que perdieron su posibilidad de ingresar nuevamente al Congreso, eran precisamente de la Nueva Mayoría.

Por otro lado, el Frente Amplio fue el principal ganador en el estreno de la reforma electoral. La falta de recursos y capacidad territorial propia de un movimiento político incipiente forzó a esta coalición a ser más selectiva y eficiente en la elección de los territorios a competir. Es más, la competencia en distritos de magnitudes altas (como el distrito 10) les permitió incluso elegir tres escaños producto de la alta votación del ex líder estudiantil y diputado del periodo 2014-2018, Giorgio Jackson.

En cambio, dentro de la centroderecha hubo ganadores y perdedores. Renovación Nacional irrumpió como el partido con mayor cantidad de escaños (36), ganando esa posición a la Unión Demócrata Independiente, que se transformó en la segunda mayor fuerza con 31 escaños de diputados. En tanto, una nueva fuerza se hizo presente en Chile Vamos. Con seis diputados y dos senadores, Evópoli se ganó un lugar dentro de los partidos tradicionales de derecha. La siguiente tabla muestra la composición de ambas cámaras luego de la elección de noviembre. 
Tabla 5. Comparación de la Cámara de Diputados y del Senado (20142018/2018-2022)

\begin{tabular}{|c|c|c|c|c|}
\hline \multirow[b]{3}{*}{ Partido } & \multirow{2}{*}{\multicolumn{2}{|c|}{ Diputados }} & & \\
\hline & & & \multicolumn{2}{|c|}{ Senado } \\
\hline & $2012-2018$ & 2018-2022 & 2012-2018 & 2018-2022 \\
\hline Partido Comunista (PC) & 6 & 8 & & \\
\hline Partido Socialista (PS) & 16 & 19 & 6 & 7 \\
\hline $\begin{array}{l}\text { Partido Movimiento Amplio } \\
\text { Social (MAS) }\end{array}$ & & & 1 & \\
\hline PAIS & & & & 1 \\
\hline Izquierda Ciudadana (IC) & 1 & & & \\
\hline Revolución Democrática (RD) & 1 & 10 & & 1 \\
\hline Partido por la Democracia (PPD) & 14 & 8 & 6 & 7 \\
\hline $\begin{array}{l}\text { Partido Radical Social Demócrata } \\
\text { (PRSD) }\end{array}$ & 6 & 8 & & 1 \\
\hline $\begin{array}{l}\text { Partido Demócrata Cristiano } \\
\text { (PDC) }\end{array}$ & 20 & 13 & 7 & 6 \\
\hline Partido Liberal de Chile (PL) & 1 & 2 & & \\
\hline Amplitud & 2 & & & \\
\hline Evópoli & 1 & 6 & & 2 \\
\hline Independientes & 8 & 1 & 5 & 1 \\
\hline Renovación Nacional (RN) & 15 & 36 & 6 & 8 \\
\hline $\begin{array}{l}\text { Unión Demócrata Independiente } \\
\text { (UDI) }\end{array}$ & 29 & 31 & 7 & 9 \\
\hline $\begin{array}{l}\text { Federación Regionalista Verde } \\
\text { Social }\end{array}$ & & 4 & & \\
\hline Partido Humanista & & 5 & & \\
\hline Partido Ecologista Verde & & 1 & & \\
\hline Partido Igualdad & & 1 & & \\
\hline Poder & & 1 & & \\
\hline Partido Progresista & & 1 & & \\
\hline Total & 120 & 155 & 38 & 43 \\
\hline
\end{tabular}

Fuente: Arana (2017) y www.servel.cl 


\section{Las elecciones presidenciales}

a) Primera vuelta

La primera vuelta de la elección presidencial enfrentó a ocho candidatos. Con una oferta programática muy diversa, el país tuvo la oportunidad de elegir entre candidatos que representaban un crisol de izquierda a derecha con nombres como Eduardo Artés, Alejandro Navarro, Marco Enriquez-Ominami, Beatriz Sánchez, Alejandro Guillier, Carolina Goic, Sebastián Piñera y José Antonio Kast.

Si bien las encuestas fueron bastante cuestionadas por errores en las predicciones, estas constantemente dieron como posibles candidatos para la segunda vuelta a tres de ocho candidatos: Sebastián Piñera (Chile Vamos), Alejandro Guillier (Fuerza de Mayoría) y Beatriz Sánchez (Frente Amplio).

Piñera, expresidente de la República durante el periodo 2010-2014, era el favorito en esta vuelta producto de la dispersión de los candidatos en la izquierda. Como favorito, la estrategia de primera vuelta fue alejarse de posibles focos de confrontación con otros candidatos y aparecer en momentos justos. Guillier, en cambio, sufría el desgaste de mantener los niveles de popularidad de principios de año luego de las disputas de la Nueva Mayoría por lograr el apoyo a su candidatura. El rostro más fresco de los tres fue Beatriz Sánchez. Con un comienzo dubitativo, la imagen de Beatriz fue paulatinamente reforzándose como una opción viable para pasar a segunda vuelta a pesar de que un mes antes la encuesta del Centro de Estudio Públicos la proyectara con un 8,6\% de los votos.

Los resultados dieron la razón a las encuestas en los tres primeros lugares mas no en los resultados. Por un lado, Sebastián Piñera obtuvo menos votos de los proyectados $(36,64 \%)$ y Beatriz Sánchez superó el umbral del veinte por ciento con un resultado del 20,27\%. Alejandro Guillier obtuvo el segundo lugar muy cerca de la candidata del Frente Amplio con un 22,7\%. Para la segunda vuelta de diciembre quedaron Alejandro Guillier y Sebastián Piñera. La tabla número 6 muestra los resultados finales de la elección. 
Tabla 6. Resultados Primera Vuelta Presidencial

\begin{tabular}{lcc}
\hline \multicolumn{1}{c}{ Nombre de Los Candidatos } & Votos & Porcentaje \\
\hline 1. Carolina Goic Boroevic & 387.780 & $5,88 \%$ \\
2. José Antonio Kast Rist & 523.213 & $7,93 \%$ \\
3. Sebastián Piñera Echenique & 2.417 .216 & $36,64 \%$ \\
4. Alejandro Guillier Álvarez & 1.497 .116 & $22,70 \%$ \\
5. Beatriz Sánchez Muñoz & 1.336 .824 & $20,27 \%$ \\
6. Marco Enríquez-Ominami Gumucio & 376.471 & $5,71 \%$ \\
7. Eduardo Artes Brichetti & 33.690 & $0,51 \%$ \\
8. Alejandro Navarro Brain & 24.019 & $0,36 \%$ \\
Válidamente Emitidos & 6.596 .329 & $98,44 \%$ \\
Votos Nulos & 65.020 & $0,97 \%$ \\
Votos en Blanco & 39.397 & $0,59 \%$ \\
\hline
\end{tabular}

Fuente: www.servel.cl

b) Segunda vuelta

La segunda ronda activó en terreno y en negociaciones a ambos candidatos. Producto de que los dos estuvieron muy lejos de la mayoría absoluta, sus estrategias se desplegaron en la conquista de los votos cercanos ideológicamente y en recomponer relaciones políticas con quienes fueron sus competidores tanto en primarias como en la primera vuelta.

Sebastián Piñera, por ejemplo, debió reunirse con Manuel José Ossandón para conquistar los esquivos votos del sur oriente de Santiago. Tras una tensa reunión entre los candidatos y los equipos, Ossandón comprometió el apoyo a Sebastián Piñera ofreciendo trabajo en la calle y en sectores populares, precisamente los lugares en que el mensaje de Piñera no había llegado con toda la fuerza necesaria. Sobre este punto Ossandón sostuvo: “...voy a estar en la calle y en los medios explicando lo que vamos a hacer como gobierno" (24 horas 2017b). 
Otro actor político importante para Piñera fue José Antonio Kast. Con un discurso de extrema derecha, Kast logró conquistar al 7,83\% de los votos. En este caso, el respaldo del excandidato a la presidencia fue inmediato, pues se adelantó en señalar que sus ideas llevarían a Sebastián Piñera al triunfo (24 horas 2017a). Con el apoyo de un candidato de derecha y otro de zonas populares, los últimos días de campaña se enfocaron en conquistar el centro político y desplazar a su adversario hacia la izquierda. Esta estrategia, de acuerdo con los resultados electorales, tuvo el efecto esperado.

En la otra vereda, la tarea de Alejandro Guillier asomaba mucho más compleja. Si bien recibió los apoyos de Marco Enríquez-Ominami y Carolina Goic, este último fue en medio de una tormenta de la Democracia Cristiana que terminó con la renuncia de la candidata a la presidencia del partido. En consecuencia, el desafío de Guillier estuvo en conquistar el 20\% de los votos que obtuvo Beatriz Sánchez, desafío mayor en especial porque las declaraciones de la candidata no explicitaron coincidencias programáticas con el proyecto del candidato de la centroizquierda. Es más, la frase con mayor resonancia de Beatriz Sánchez en segunda vuelta se produjo cuando, luego de dar un "apoyo personal" a la candidatura de Guillier, señaló no sentirse "dueña de los votos de las personas".

Con todo, esta difícil tarea no logró los objetivos. En el cierre de campaña se invitó a José "Pepe" Mujica, expresidente uruguayo, figura importante que no alcanzó para las pretensiones del oficialismo de continuar con el legado de Bachelet. El resultado final de la segunda vuelta fue de 54,58\% para Piñera y $45,42 \%$ para Guillier, amplia diferencia que superó las expectativas de muchos personeros de ambas coaliciones.

\section{DISCUSIÓN}

El año político 2017 en Chile estuvo centrado en las elecciones. En estas, los candidatos y partidos debieron enfrentar nuevas reglas del juego tanto en temas de financiamiento electoral como en campañas y reglas electorales.

Ante el nuevo escenario, la incertidumbre primó en muchos actores políticos. Esa incertidumbre fue absorbida de distintas maneras. En este repaso se describió cómo los parlamentarios (especialmente incumbentes) tomaron decisiones en relación con sus posibilidades, optando principalmente por tres conductas a) la ambición discreta, b) la ambición estática y c) la ambición progresiva. Esas decisiones llevaron a consecuencias en las estrategias y expectativas. Para enfrentar distritos más grandes se utilizó la segmentación territorial dentro de los pactos, a través de la división de sectores entre compañeros de lista. En este artículo demostramos que las votaciones de los candidatos en cada comuna no fueron homogéneas y dependieron mucho de la capacidad territorial de cada uno de los candidatos. 
Por otro lado, en términos de expectativas, el sistema electoral abrió el camino para nuevos grupos políticos. Por ejemplo, políticos de base territorial como consejeros regionales y alcaldes pudieron alcanzar un escaño. De la misma manera, más mujeres fueron a la competencia política, así como nuevos partidos o movimientos fueron alternativas políticas viables.

Con todo, los resultados de las elecciones han generado diferentes reacciones entre analistas, políticos e incluso académicos. Falta mucho por saber qué ocurrió realmente con el cambio de las reglas electorales. Hasta ahora, quedan más preguntas que respuestas. ¿Qué pasa con la base territorial de los incumbentes? ¿Cómo entran al juego político los nuevos representantes subnacionales? ¿Cuánto sigue pesando el trabajo de terreno? ¿De qué forma se financiarán las campañas ante las nuevas restricciones? ¿Cuánto pesa la segmentación territorial? Falta, sin embargo, encontrar respuestas no lineales a estas preguntas. Es misión de la disciplina allanar el camino para este nuevo desafío.

\section{REFERENCIAS}

24 horas. 2017a. "Kast entrega su apoyo a Piñera." Recuperado el 2 de febrero de 2018 de http:/ / www.24horas.cl/politica/elecciones2017/jose-antonio-kast-entrega-su-respaldo-a-sebastian-pinera-para-la-segunda-vuelta-2567580

24 horas. 2017b. "Ossandón en apoyo a Piñera." Recuperado el 2 de febrero de 2018 de http:/ / www.24horas.cl/politica/ossandon-en-apoyo-a-pinera-voy-a-estar-en-la-calle-y-medios-explicando-lo-que-vamos-a-hacer-como-gobierno-2570992

24 horas. 2017c. "Piñera por campaña de Ossandón: 'Se ha quedado atrapada en muchas mentiras.'" Recuperado el 2 de febrero de 2018 de http:/ / www.24horas.cl/politica/elecciones2017/ primarias2017 / pinera-por-campana-de-ossandon-se-ha-quedado-atrapada-en-muchas-mentiras-2420817

Albertini, Camila. 2017. “La cronología que desató la renuncia del equipo económico de Bachelet." Publimetro.

Alemán, Eduardo. 2008. "Policy Positions in the Chilean Senate: An Analysis of Coauthorship and Roll Call Data." Brazilian Political Science Review 2 (2): 74-92.

Aleman, Eduardo, Juan Pablo Micozzi y Margarita Ramírez. 2016. "The Hidden Electoral Connection: Analyzing Parliamentary Questions in the Chilean Congress." The Journal of Legislative Studies 24 (2): 227-244.

Altman, David. 2016. "Informe Especial: La caída del Binominal." Cultura política de la democracia en Chile y en las Américas, 2014: ¿Tiempo de reformas?, editado por Juan Pablo Luna, Sergio Y. Toro, Nathalie Jaramillo-Brun y Carolina Acevedo. Nashville: Vanderbilt University, PUC.

Arana Araya, Ignacio. 2017. “Chile 2016: ¿El nadir de la legitimidad democrática?" Revista de Ciencia Política 37 (2): 305-334.

Bhattacharya, Mita, Sudharshan Reddy, Ilhan Ozturk y Sankar Bhattacharya. 2016. "The Effect of Renewable Energy Consumption on Economic Growth : Evidence from Top 38 Countries." Applied Energy 162: 733-741.

Boeninger, Edgardo. 2007. Políticas Públicas en Democracia. Santiago: Uqbar.

Cámara de Diputados. 2013. "Informe de la comisión de familia constituida en investigadora para recabar información y determinar responsabilidades en las denuncias sobre hechos ilícitos ocurridos en hogares del Servicio Nacional de Menores." 
Cámara de Diputados. 2017. “Informe de la Comisión Investigadora del SENAME en el año 2014."

Cámara de Diputados. 2018. “Comisiones." Recuperado el 2 de febrero de 2018 de https:// www.camara.cl/trabajamos/comision_portada.aspx?prmID=1581

Camerlo, Marcelo y Aníbal Pérez-Liñan. 2014. "The Politics of Minister Retention: Technocrats, Partisans, and Government Approval." ECPR General Conference Sciences Po, Bordeaux, septiembre.

Campos-Parra, Hernán y Patricio Navia. 2017. “Disciplina legislativa en la Cámara de Diputados de Chile, 2010-2014." Política y Gobierno 24 (1): 81-123.

CNE. 2018. Reporte Mensual ERCN. Santiago de Chile.

Domínguez, Francisca. 2016. "30-30-30: La 'extrema' combinación de números detrás del aumento de incendios forestales." Recuperado el 8 de febrero de 2018 de http:/ /www. emol.com/noticias/Nacional/2016/11/22/832203/303030-Una-de-las-razones-delaumento-de-incendios-forestales-en-la-zona-central-del-pais.html

Gamboa, Ricardo y Carolina Segovia. 2016. “Chile 2015: falla política, desconfianza y reforma." Revista de Ciencia Política 36: 123-144.

Gobierno de Chile. 2017. "Reconstrucción." Recuperado el 2 de febrero de 2018 de http:// reconstruccion.gob.cl

Ley 20840. 2015. Sustituye el sistema electoral binominal por uno de carácter proporcional inclusivo y fortalece la representatividad del Congreso Nacional. Santiago de Chile.

Martinez-Gallardo, Cecilia. 2012. "Out of the Cabinet, What Drives Defections from the Government in Presidential System?" Comparative Political Studies 45 (1): 62-90.

Martínez-Harms, María José, Hernán Cáceres, Duan Biggs y Hugh P. Possingham. 2017. “After Chile's Fires, Reforest Private Land." Science 356 (6334): 147-148.

El Mostrador. 2017. "No te declararon reo por lindo." Recuerado el 2 de febrero de 2018 de http://www.elmostrador.cl/noticias/pais/2017/06/27/no-te-declararon-reopor-lindo-frase-de-ossandon-a-pinera-se-convierte-en-trending-topic/?php bloginfo(\%27url\%27); ?\%3E/cultura

Plataforma Urbana. 2017. "Sensor térmico de la NASA."

PNUD. 2017. Representación política de mujeres en el Poder Legislativo: Análisis de la aplicación de las cuotas de género en las elecciones parlamentarias de 2017. Santiago de Chile.

Saffirio, René. 2017. "De las 1.313 muertes en el Sename, sólo se hicieron 23 autopsias." Recuperado el 8 de febrero de 2018 de https:/ / www.cnnchile.com/pais/diputado-saffirio-de-las-1313-muertes-en-el-sename-solo-se-hicieron-23-autopsias_20170710/

Schlesinger, Joseph A. 1966. Ambition and Politics: Political Careers in the United States. Chicago: Rand McNally.

Sepúlveda, Nicolás y Mónica González. 2018. "La trama de pagos de privados a Carabineros que el General Hermes Soto enfrentó." Recuperado el 2 de febrero de 2018 de https: / / ciperchile.cl/2018/03/23/la-trama-de-pagos-de-privados-a-carabineros-que-el-general-hermes-soto-enfrento/

SUBTRAB. 2018. "Cifras de empleo." Recuperado el 2 de febrero de 2018 de http:/ /www. subtrab.trabajo.gob.cl/programas-de-empleo/cifras-de-empleo/\%3E

Tele13. 2017. "Financiamiento: Aleuy llama a candidatos a 'ser creativos' y descarta Intervención Del Gobierno." Recuperado el 2 de febrero de 2018 de http:/ / www.t13.cl/radio/ politica/mesa-central/noticia/financiamiento-aleuy-llama-candidatos-ser-creativos-y-descarta-intervencion-del-gobierno

Toloza, Mackarena y Sergio Y. Toro. 2017. “Amigos cerca, enemigos más cercae El Gobierno de Sebastián Piñera y las dinámicas legislativas en Chile." Revista Uruguaya de Ciencia Política 26 (1): 131-149.

Toro, Sergio Y. 2007. "Conducta Legislativa ante las iniciativas del Ejecutivo: Unidad de los bloques políticos en Chile." Revista de Ciencia Política 27 (2): 23-41.

Toro, Sergio Y. 2017. "Lejos del Congreso: Explicando el trabajo distrital de los parlamentarios chilenos." En Percepciones y actores de la representación política en América Latina, editado por Leticia Rodriguez. Barcelona: Hoygens Editorial, 159-177. 
Sergio Toro Maureira es Doctor en Ciencia Política y Profesor Asociado del Departamento del Administración Pública y Ciencia Política de la Universidad de Concepción. Actualmente es director del Centro de Información para la Democracia, UdeC. Email: sergiotoro@udec.cl

Macarena Valenzuela Beltrán es candidata a doctora en Ciencia Política por la Universidad Diego Portales. Actualmente es investigadora del Centro de Información para la Democracia, UdeC. 